\title{
Home Intravenous Antimicrobial Infusion Therapy: A Viable Option in Older Adults
}

\author{
Amanda M. Cox, MD, * Preeti N. Malani, MD, ${ }^{\dagger+\mathcal{S}}$ Stephen W. Wiseman, PharmD, "and \\ Carol A. Kauffman, $M D^{\dagger}$
}

(See editorial comments by Dr. Kevin High on pp 792-793)

OBJECTIVES: To determine whether older adults and younger adults are equally able to administer home intravenous antimicrobial infusion therapy (home IV antimicrobials) without intensive support from home care agencies.

DESIGN: Retrospective cohort study.

SETTING: Veterans Affairs Ann Arbor Healthcare System, a 100-bed tertiary care medical center.

PARTICIPANTS: All patients who received home IV antimicrobials from July 1, 2000, through December 31, 2003.

MEASUREMENTS: Demographic data, underlying medical conditions, indications for therapy, antimicrobial agents administered, concomitant medications, frequency of patient visits and phone calls, adverse events, and outcomes of infections.

RESULTS: A total of 205 patients received 231 courses of home IV antimicrobials, with 107 courses in patients aged 60 and older and 124 courses in patients younger than 60 . For both groups, the most common indication for therapy was osteoarticular infections, and the predominant pathogens were Staphylococcus aureus and coagulase-negative Staphylococcus. Older patients were significantly more likely than younger patients to require the assistance of family members to help with the infusion and were more likely to be seen in urgent care or to call the infectious diseases pharmacist or physicians with questions. Overall, clinical outcomes and numbers of adverse events were similar in both groups, with the exception of nephrotoxicity, which was greater in the older group $(P=.02)$.

CONCLUSION: With appropriate support from a hospital-based home IV antimicrobials therapy team, home IV

From the Divisions of * General Medicine, Infectious Diseases, and ${ }^{\ddagger}$ Geriatric Medicine, Department of Internal Medicine, ${ }^{\S}$ Geriatric Research Education and Clinical Center, and "Department of Pharmacy Services, Veterans Affairs Ann Arbor Healthcare System, University of Michigan Health System, Ann Arbor, Michigan.

Address correspondence to Dr. Preeti N. Malani, VA Healthcare System, 2215 Fuller Road, Ann Arbor, MI 48105. E-mail: pmalani@umich.edu

DOI: 10.1111/j.1532-5415.2007.01133.x antimicrobial appears to be a viable option for older adults. J Am Geriatr Soc 55:645-650, 2007.

Key words: elderly; older adults; home infusion; antimicrobial agents

Serious infections often require extended courses of intra$\checkmark$ venous antimicrobial agents. Community-based or home intravenous antimicrobial infusion therapy (home IV antimicrobials) provides a convenient and cost-effective means to complete extended courses of antimicrobial agents. Increasingly, these agents are administered in the home setting for many patients, including older adults. ${ }^{1-6}$ Few studies have specifically addressed concerns unique to the older adult who receives home IV antimicrobials. ${ }^{7}$ One report noted that $72 \%$ of older adults were rejected as candidates for home IV antimicrobials, compared with a rejection rate of $49 \%$ for patients of all ages. ${ }^{1}$ Concern exists that older adults, who often have more comorbid conditions and functional impairment, might be at risk for more adverse events while administering home IV antimicrobials. In the private sector, hiring agencies that specialize in home infusion therapy to oversee the administration of antimicrobials to older adults can often allay this concern.

The Veterans Affairs Ann Arbor Healthcare System differs from many in the private sector in that it does not contract with home care agencies to supply antimicrobial agents, perform infusions, or monitor laboratories. Patients and caregivers are taught the techniques required for administration of home IV antimicrobials, all drugs and supplies are provide through the pharmacy, and adverse events and clinical outcomes are monitored in the infectious diseases clinic. At every step, this is accomplished with the help of a dedicated infectious diseases pharmacist who administers the home IV antimicrobials program (SWW).

The objective of the study was to determine whether older adults were able to successfully administer home 
IV antimicrobials without intensive support from home care agencies. The occurrence of adverse events and outcomes in all patients in the home IV antimicrobials program was studied, and whether older patients had worse outcomes and more adverse events than younger patients was specifically determined.

\section{METHODS}

\section{Setting and Patients}

The Veterans Affairs (VA) Ann Arbor Healthcare System is a 100-bed tertiary care medical center. The study population included all patients who received home IV antimicrobials during July 1, 2000, to December 31, 2003. Study patients were identified using a computerized database maintained by the infectious diseases pharmacist. All patients had a long-term venous access device (a peripherally inserted central catheter (PICC), a tunneled semipermanent central catheter, or an infusion port). Laboratory studies were monitored when the patients returned to the clinic, which was generally every 2 weeks but more often if required by the clinical situation. The institutional review board of the VA Ann Arbor Healthcare System approved the study.

\section{Overview of the Home IV Antimicrobials Program}

The infectious diseases consult team, the infectious diseases pharmacist, and the inpatient IV therapy team saw patients who were to be discharged from hospital and receive home IV antimicrobials. Most patients had PICC lines placed before discharge; tunneled central venous catheters and infusion ports were rarely used unless there was another indication, such as a requirement for chemotherapy or parenteral nutrition. Before discharge, a member of the IV therapy team, who verified that a patient could safely perform IV infusions at home before the patient was discharged, instructed patients and their families or other caregivers in infusion techniques. Visiting nurses were available to change the dressing over the catheter insertion site each week for some patients; others returned to the infectious diseases clinic weekly to have the dressing changed. The pharmacist tracked the number of calls to the infectious diseases pharmacist, nurse case manager, and physicians and the number of urgent care visits to the facility or other facilities related to questions about the catheter or infusion.

\section{Study Design and Data Collection}

This retrospective review evaluated the pharmacist's records, patients' medical records, dispensing information from the pharmacy, and microbiology data. For each patient receiving home IV antimicrobials, the infectious diseases pharmacist prospectively recorded antimicrobial agents dispensed, details of the IV catheter and insertion site, adverse events, and overall outcome. Information collected for this analysis included demographic data, underlying medical conditions, indications for antimicrobial therapy, specific antimicrobial agents administered, concomitant medications, frequency of patient visits and laboratory testing, adverse events, admissions to hospital or visits to urgent care for problems related to the catheter or adverse events, and outcome of the infection.

\section{Definitions of Adverse Events}

The following adverse events were recorded: allergic reactions (rash, urticaria, anaphylaxis); nephrotoxicity (increase in serum creatinine of $\geq 1 \mathrm{mg} / \mathrm{dL}$ or a doubling of serum creatinine from with the baseline value on the first day of outpatient therapy); leukopenia (white blood cell count $<4,000 / \mathrm{uL}$ ); neutropenia (neutrophils $<500 / \mathrm{uL}$ ); anemia (hemoglobin $<10 \mathrm{mg} / \mathrm{dL}$ ); thrombocytopenia (platelets $<100,000 / \mathrm{uL}$ ); and eosinophilia (eosinophils $>500 / \mathrm{uL}$ ). Venous access device complications that the record was reviewed for were deep venous thrombosis, phlebitis, accidental removal of the device by the patient, leaking, local infection, and device-related bloodstream infection.

\section{Statistics}

Values were expressed as means \pm standard deviations. For adverse events, data were expressed per 1,000 home IV antimicrobials days. Chi-square, chi-square with continuity correction, and Fisher exact tests were used to compare proportions. A two tailed $P$-value $<.05$ was considered significant.

\section{RESULTS}

\section{Patient Characteristics}

A total of 279 patients who underwent 307 courses of IV antimicrobial therapy were evaluated for home IV antimicrobials during the 3.5 years of the study; this included 147 courses of antimicrobial therapy in the older age group $(\geq 60)$ and 160 courses in the younger age group $(<60)$. There were 40 courses in patients aged 60 and older and 36 courses in patients younger than 60 that were not given as home IV antimicrobials. In the older group, the predominant reasons for excluding patients were that they were deemed incapable of performing the infusion (19/147, $13 \%)$ and the IV antimicrobial agent was no longer needed $(14 / 147,10 \%)$. In the younger group, the predominant reasons for exclusion were that the IV antimicrobial agent was no longer needed $(18 / 160,11 \%)$ and that they were deemed incapable of performing the infusion $(8 / 160,5 \%)$. A small number of patients in both groups were excluded, because they were transferred to another facility or they stayed in the extended care facility to undergo rehabilitation along with IV antimicrobial therapy.

A total of 205 patients received 231 courses of home IV antimicrobials during July 1, 2000, to December 31, 2003. Thirteen patients received two separate courses of home IV antimicrobials, and six patients received three or more courses of home IV antimicrobials. The overall mean age was $58.8 \pm 12.2$ (range 23-83). Of those aged 60 and older, the mean age was $68.9 \pm 8.9$, and 94 patients $(46 \%)$ received 107 courses of home IV antimicrobials; of those younger than 60 , the mean age was $50.6 \pm 8.1$, and 111 patients $(54 \%)$ received 124 courses of home IV antimicrobials. Not unexpectedly in a VA population, $99 \%$ of patients were men; there were two women, both younger than 60 . The number of home IV antimicrobials courses increased during the study period; there were 28 courses in the second half of 2000,52 in 2001, 72 in 2002, and 79 in 2003.

Underlying medical illnesses were common in younger and older adults. The most common underlying illnesses 
Table 1. Underlying Conditions of 205 Patients Receiving 231 Courses of Home Intravenous Antimicrobials

\begin{tabular}{|c|c|c|c|}
\hline \multirow[b]{2}{*}{ Underlying Condition* } & Aged $\geq 60(n=107)$ & Aged $<60(n=124)$ & \multirow[b]{2}{*}{$P$-value } \\
\hline & \multicolumn{2}{|c|}{ Courses, n (\%) } & \\
\hline Coronary artery disease & $66(62)$ & $41(33)$ & .01 \\
\hline Diabetes mellitus & $62(58)$ & $63(51)$ & .34 \\
\hline Neuropathy & $36(34)$ & $42(34)$ & .92 \\
\hline Peripheral vascular disease & $38(36)$ & $29(23)$ & .06 \\
\hline Congestive heart failure & $33(31)$ & $18(15)$ & .01 \\
\hline Surgery in prior month & $19(18)$ & $16(13)$ & .40 \\
\hline Chronic renal insufficiency & $18(17)$ & $12(10)$ & .16 \\
\hline Malignancy & $17(16)$ & $19(15)$ & .95 \\
\hline Spinal cord injury & $7(7)$ & $8(7)$ & .81 \\
\hline Stroke & $4(4)$ & $6(5)$ & .94 \\
\hline Cirrhosis & $3(3)$ & $2(2)$ & .86 \\
\hline
\end{tabular}

${ }^{*}$ Most patients had more than one underlying condition.

were diabetes mellitus, coronary artery disease, neuropathy, and peripheral vascular disease (Table 1$)$. Coronary artery disease $(P=.01)$ and congestive heart failure $(P=.01)$ were significantly more common in patients aged 60 and older.

\section{Indications for Home IV Antimicrobials}

The most common indications for therapy were osteoarticular infections $(52 \%)$, bacteremias $(14 \%)$, and skin and soft-tissue infections (13\%) (Table 2). A significant difference in indications between older and younger age groups was not observed for home IV antimicrobials, although there was a trend toward more abdominal abscesses in the younger group $(P=.06)$ and more genitourinary tract infections in the older group $(P=.08)$.

The total days of home IV antimicrobials was 2,643 for the older adults group and 4,303 for the younger group. The mean duration of therapy for older adults was $24.7 \pm 17$ days (median 22 days; range $2-105$ days) and for younger adults was $34.7 \pm 42.3$ (median 29.5 days; range 4-450 days). The difference in mean days of therapy stems partly from one patient in the younger group who received 450 days of amphotericin $\mathrm{B}$ for meningitis and lumbar epidural abscess due to Coccidioides immitis. With- out this patient, the mean for the younger group was 31.3 days.

The predominant pathogen for which home IV antimicrobials were used was Staphylococcus aureus (39\% of all episodes), followed by coagulase-negative Staphylococcus (23\%), Enterococcus faecalis (14\%), and Pseudomonas aeruginosa (13\%). Of the S. aureus isolates, $28(26 \%)$ were methicillin resistant (MRSA) in the older group and $14(11 \%)$ in the younger group $(P=.005)$. There were more gram-negative bacilli found in younger patients than in older patients $(53(43 \%)$ vs $29(27 \%), P=.02)$. There were no other significant differences found in organisms infecting older and younger patients. In $18(17 \%)$ older patients and $26(23 \%)$ younger patients, almost all of whom had osteomyelitis, the etiologic agent was not known, and emperical antimicrobial treatment was given.

\section{Antimicrobial Therapy}

The most common antimicrobial agent for home IV antimicrobials was vancomycin, which was given for $46 \%$ of all episodes. Cefazolin, ceftriaxone, and ertapenem constituted the majority of the remaining antimicrobial agents. Aminoglycosides were used infrequently (Table 3 ). Older pa-

Table 2. Indications for Home Intravenous Antimicrobials in 205 Patients Receiving 231 Courses of Therapy

$$
\text { Aged } \geq 60(n=107) \quad \text { Aged }<60(n=124)
$$

Type of Infection*

Courses, n (\%)

$P$-value

\begin{tabular}{lccc}
\hline Osteoarticular & $56(52)$ & $64(52)$ & .98 \\
Bacteremia & $17(16)$ & $15(12)$ & .52 \\
Cellulitis, subcutaneous abscess & $14(13)$ & $15(12)$ & .99 \\
Surgical site infection & $8(8)$ & $6(5)$ & .57 \\
Endocarditis & $7(7)$ & $8(7)$ & .99 \\
Genitourinary infection & $6(6)$ & $1(1)$ & .08 \\
Respiratory tract infection & $5(5)$ & $7(6)$ & .98 \\
Abdominal abscess & $1(1)$ & $8(7)$ & .06 \\
Other & $2(2)$ & $5(4)$ & .58
\end{tabular}

* Several courses were associated with more than one infection.

${ }^{\dagger}$ Neurosyphilis, Coccidioides meningitis, toxoplasmosis, spontaneous bacterial peritonitis, and pericardial abscess. 
Table 3. Antimicrobial Agents Given as Home Intravenous Antimicrobials in 231 Courses in 205 Patients

\begin{tabular}{lccc}
\hline & \multicolumn{2}{c}{ Courses, $\mathrm{n}(\%)$} & \\
\cline { 2 - 3 } & $\begin{array}{c}\text { Aged } \geq 60 \\
\text { Antimicrobial Agent* }\end{array}$ & $\begin{array}{c}\text { Aged <60 } \\
(\mathrm{n}=107)\end{array}$ & \\
\hline Vancomycin & $58(54)$ & $47(38)$ & .02 \\
Ertapenem & $13(12)$ & $17(14)$ & .88 \\
Cefazolin & $11(10)$ & $17(14)$ & .55 \\
Ceftriaxone & $10(9)$ & $14(11)$ & .79 \\
Ceftazidime & $12(11)$ & $4(3)$ & .03 \\
Piperacillin-tazobactam & $6(6)$ & $8(7)$ & .99 \\
Ampicillin-sulbactam & $4(4)$ & $13(11)$ & .08 \\
Aminoglycosides & $2(2)$ & $9(7)$ & .10 \\
Other agents ${ }^{\dagger}$ & $7(7)$ & $18(15)$ & .08 \\
\hline
\end{tabular}

* Some courses involved treatment with more than one agent.

${ }^{\dagger}$ Clindamycin, penicillin, nafcillin, ampicillin, ticarcillin-clavulanic acid, imipenem, meropenem, and amphotericin B.

tients were more likely than younger patients to be treated with vancomycin $(P=.02)$ and ceftazidime $(P=.03)$.

Concomitant medications did not differ significantly between older and younger patients. Forty-six patients in each group received angiotensin-converting enzyme inhibitors, and $54(51 \%)$ older adults and $33(27 \%)$ younger adults received diuretics $(P=.06)$. Nonsteroidal antiinflammatory agents were used in 12 patients $(11 \%)$ in the older group and 18 patients $(15 \%)$ in the younger group.

\section{Intravenous Catheters and Their Care}

Overall, 220 of the 231 courses of home IV antimicrobials were given through a PICC. Five patients in the older group and five patients in the younger group had a peripheral catheter (nonindwelling) used for short courses of home IV antimicrobials, three patients had midline catheters placed, and four patients each had an infusion port or a Hickman tunneled catheter. For nine courses, patients required placement of more than one device. No infusion pumps were used to administer antimicrobials; all patients used gravity infusion techniques.

For 72 of $231(31 \%)$ courses of home IV antimicrobials, the patient performed the infusions without the help of others. The younger group was significantly more likely to do this $(51 / 124$ episodes, $41 \%)$, than the older group (21/107 episodes, $20 \%)(P<.001)$. More commonly in the older group, family members or friends performed the infusions $(37 / 107$ courses $(35 \%)$ vs $28 / 124$ courses $(23 \%)$ in the younger group, $P=.03)$. The patient, in concert with a family member or friend, was responsible for infusions in 30 courses $(28 \%)$ in the older group and 36 courses $(29 \%)$ in the younger group. For 10 courses $(9 \%)$ in older patients and three courses $(2 \%)$ in younger patients, a visiting nurse was available to help with the infusion. A variety of other arrangements, including urgent care visits for infusions and varying combinations of family and friends, made up the remainder. The patient or the family performed daily inspection of the catheter insertion site; a visiting nurse changed the catheter site dressing once weekly for $78(73 \%)$ courses in older patients and $59(47 \%)$ courses in younger patients $(P<.001)$. In the remainder of the patients, family members, a nearby VA clinic, or the infectious diseases clinic performed dressing changes.

\section{Adverse Events}

Nephrotoxicity was observed in 10 courses of home IV antimicrobials. There was more nephrotoxicity in the older group (3.0/1,000 home IV antimicrobials days) than in the younger group $(0.5 / 1,000$ home IV antimicrobials days) $(P=.02)$ (Table 4). Other adverse events occurred no more frequently in the older group than in the younger group (Table 4). Venous access device complications were frequent but rarely serious. The most common event was occlusion of a PICC line, which occurred at a rate of 3.0 per 1,000 home IV antimicrobials days in older patients and 2.3 per 1,000 home IV antimicrobials days in younger patients

Table 4. Adverse Events Noted in 231 Courses of Home Intravenous (IV) Antimicrobials in 205 Patients

\begin{tabular}{lccc}
\hline & Aged $\geq 60(\mathrm{n}=107)$ & Aged $<60(\mathrm{n}=124)$ \\
\cline { 2 - 4 } \multicolumn{1}{c}{ Adverse Event } & $\mathrm{n}($ Rate/1,000 Home IV Antimicrobials Days) & $P$-value \\
\hline Rash & $4(1.51)$ & $4(0.93)$ & .74 \\
Nephrotoxicity & $8(3.03)$ & $2(0.46)$ & .02 \\
Eosinophilia & $4(1.51)$ & $5(1.16)$ & .96 \\
Leukopenia & $2(0.76)$ & $5(1.16)$ & .96 \\
Anemia & $3(1.14)$ & $2(0.46)$ & .58 \\
Thrombocytopenia & $3(1.14)$ & 0 & .08 \\
Occlusion of catheter & $8(3.03)$ & $10(2.32)$ & .75 \\
Catheter pulled out & $9(3.41)$ & $13(3.02)$ & .95 \\
Irritation from dressing & $2(0.76)$ & $4(0.93)$ & .86 \\
Site infection & $2(0.76)$ & $2(0.46)$ & .98 \\
Leakage & $1(0.38)$ & $2(0.46)$ & .67 \\
Bacteremia & $2(0.76)$ & $1(0.23)$ & .67 \\
Phlebitis & 0 & $1(0.23)$ & .62 \\
Deep venous thrombosis & 0 & 0 & 0 \\
\hline
\end{tabular}


$(P=.7)$; these events resolved with fibrinolytic agents and did not require catheter removal. One patient in the younger group developed bacteremia with $S$. aureus related to a Hickman catheter, and one older patient each developed Candida parapsilosis fungemia and coagulase-negative Staphylococcus bacteremia associated with a PICC line. In all three cases, the catheters were removed, and the infections were successfully treated with antimicrobial agents. Catheter or infusion problems resulted in hospital admission during three courses in older patients and two courses in younger patients.

The number of calls to the infectious diseases team and the number of urgent care visits related to questions about the catheter or the infusion differed between older and younger patients. Older patients were significantly more likely than younger adults to have a visit to urgent care with a problem or question related to home IV antimicrobials (31.4 visits/1,000 home IV antimicrobials days vs 14.3 visits/1,000 home IV antimicrobials days, respectively, $P<.001)$. Older adults were also more likely than younger adults to need intervention from social work service (7.1 encounters/1,000 home IV antimicrobials days vs 2.9 encounters/1,000 home IV antimicrobials days, respectively, $P=.01)$. Finally, there were more phone calls to the infectious diseases pharmacist from the older group (14.3/1,000 home IV antimicrobials days) than from the younger group (8.57/1,000 home IV antimicrobials days) $(P=.04)$.

\section{Outcomes}

A total of 27 courses in older patients $(25 \%)$ and 27 courses in younger patients $(22 \%)$ resulted in cure of the infection by the end of home IV antimicrobials. Overall, 70 courses in older patients $(65 \%)$ and 89 courses in younger patients $(72 \%)$ were deemed to have stable or improved infection at the conclusion of intravenous therapy. Oral antimicrobial therapy, mostly for osteoarticular and complicated skin and soft-tissue infections, was given when home IV antimicrobials were finished in patients with stable and improved infection. In only nine courses in older adults $(8 \%)$ and seven courses in younger adults $(6 \%)$ was home IV antimicrobials considered a failure, and in all of these cases, failure was attributed to a difficult-to-treat infection and not to adverse effects encountered during home IV antimicrobials.

\section{DISCUSSION}

During the past 2 decades, the use of home IV antimicrobials has increased greatly. With improving support networks and growing pressure to decrease hospital lengths of stay, an increasing number of patients are sent home to complete their course of antimicrobial therapy. However, many older individuals still require transfer to a skilled care facility to receive antimicrobial therapy. This occurs in part because of concerns that older adults might have more difficulty with the fine manipulations needed to perform the infusions or may be unable to understand the nuances of sterile technique and flushing of the catheter. ${ }^{7}$ However, many older adults would prefer to go home than to a skilled care facility. Given the predominance of older patients served by the VA healthcare system and the fact that this system provides total care for most of its patients, this study was in a unique position to compare the effectiveness and safety of home IV antimicrobials in a large group of older adults with that in a younger cohort.

In the home IV antimicrobials program, patients and their families assume responsibility for storage and mixing of the antimicrobial agents, infusion of the drug, flushing of the catheter, and daily observation of the catheter site. The program is managed through the infectious diseases clinic and the infectious diseases pharmacist, rather than a home care agency. For some patients, it was possible to obtain help from a visiting nurse for a limited number of infusions. Patients or families who have questions or problems have easy access to the infectious diseases clinic team, which includes the pharmacist, the nurse care manager, physicians, and a social worker.

This study found that both groups experienced fewer adverse events related to antimicrobial agents than noted in several previous studies. ${ }^{2,8-12}$ For example, only eight older adults and two younger adults experienced nephrotoxicity during 231 courses of therapy. This may relate partly to the fact that aminoglycosides were used in 11 patients, of whom only two were aged 60 and older. Other reports have included a higher proportion of patients receiving aminoglycosides as home IV antimicrobials. ${ }^{8}$ Bone marrow toxicity was also infrequently noted and was most often related to the use of beta-lactam agents. Rash, which was always associated with the administration of a beta-lactam antibiotic, was noted during only four courses in patients in each age group.

In this study, adverse events related to the catheter were no different between the age groups, and few were serious. Only three bloodstream infections were noted, which is less than noted in other reports. ${ }^{11}$ This is likely related to the fact that the patients were not receiving parenteral nutrition and few were immunosuppressed, which are risk factors for catheter-related bloodstream infection in patients receiving home infusion therapy. ${ }^{11}$ Occlusion of PICCs was common but resolved in all patients with the use of fibrinolytic therapy. Deep venous thrombosis was not seen in either group. This is a serious complication of PICC placement and is likely underdiagnosed. ${ }^{9}$ Although it is possible that this complication was missed, few of the patients had the risk factors found in a previous study to contribute to development of great vein thrombosis, namely amphotericin B administration, prior history of thrombotic events, and care in a long-term care facility. ${ }^{9}$

This review identified a few differences between the older and younger age groups. The differences that were noted related almost entirely to the support needed to perform the infusions and care for the catheter. In only $20 \%$ of courses in older patients did the patient perform the infusion entirely; most older patients needed a family member to perform the infusion or to help with certain aspects of administering the antimicrobial agents. In many cases, this caregiver was a spouse, who was generally similar in age to the patient. Older patients also were significantly more likely than younger patients to call the infectious diseases team or go to urgent care for advice about the catheter or the infusion itself.

This study has several potential limitations. It is possible that older adults who were most adept at understanding the instructions and most facile at performing the 
infusions were predominantly selected. Patients who required other types of care, such as complicated wound dressings, were treated in the attached skilled care facility rather than being offered home IV antimicrobials. Also, all patients had to perform at a level deemed appropriate by the IV therapy team before they were able to go home; this likely selected for more-functional older adults. Whereas $13 \%$ of older adults or their caregivers were deemed incapable of performing the tasks required for home IV antimicrobials, $5 \%$ of younger adults or their caregivers were considered incapable during the study period. Additionally, it is possible that some patients called their local physician with questions about the infusion and that there was therefore not a record of all of these calls, although it is unlikely that many calls were missed, because the vast majority of the patients obtain their primary care from the VA system and do not have a private physician.

The results show that home IV antimicrobials is a viable and safe option for older patients. Family members play an important role in allowing older relatives to come home from the hospital earlier and to continue to receive appropriate antimicrobial therapy. As emphasized in the Infectious Diseases Society of America Guidelines for Outpatient Parenteral Antimicrobial Therapy ${ }^{6}$ assessment of the patient's and the caregiver's abilities to perform home infusion is crucial to the success of any home infusion program. There have been only a few problems with the patient or the caregiver being unable to perform the infusions after discharge. Use of the resources of the VA Healthcare System to support home IV antimicrobials is effective for care of older and younger adults.

\section{ACKNOWLEDGMENTS}

Financial Disclosure: This work was supported in part by an educational grant from Astellas Pharmaceuticals, Inc.
Author Contributions: Cox and Malani: collection and analysis of data, manuscript preparation. Wiseman: acquisition of subjects and collection of data, manuscript preparation. Kauffman: study design, collection and interpretation of data, manuscript preparation.

Sponsor's Role: None; authors retained complete independence in scientific investigation and reporting.

\section{REFERENCES}

1. Rehm SJ, Weinstein AJ. Home intravenous antibiotic therapy: A team approach. Ann Intern Med 1983;99:388-392.

2. Rehm SJ, Longworth DL. Rates of adverse events associated with communitybased parenteral anti-infective therapy. J Clin Outcomes Manag 2000;7: 23-28.

3. Tice AD, Hoaglund PA, Shoultz DA. Risk factors and treatment outcomes in osteomyelitis. J Antimicrob Chemother 2003;51:1261-1268.

4. Andrews MM, von Reyn CF. Patient selection criteria and management guidelines for outpatient parenteral antibiotic therapy for native valve endocarditis. Clin Infect Dis 2001;33:203-209.

5. Gilbert DN, Dworkin RJ, Raber SR et al. Outpatient parenteral antimicrobial drug therapy. N Engl J Med 1997;337:829-838.

6. Tice AD, Rehms SJ, Dalovisio JR et al. Practice guidelines for outpatient parenteral antimicrobial therapy. Clin Infect Dis 2004;38:1651-1672.

7. Chattopadhyay T, Catania PN, Mergener MA. Therapeutic outcome of elderly and nonelderly patients receiving home intravenous antimicrobial therapy. Am J Hosp Pharm 1990;47:335-339.

8. Hoffman-Terry ML, Fraimow HS, Fox TR et al. Adverse effects of outpatient parenteral antibiotic therapy. Am J Med 1999;106:44-49.

9. Chemaly RF, Parres JB, Rehm SJ et al. Venous thrombosis associated with peripherally inserted central catheters. A retrospective analysis of the Cleveland Clinic experience. Clin Infect Dis 2002;34:1179-1183.

10. Wynn M, Dalovisio JR, Tice AD et al. Evaluation of the efficacy and safety of outpatient parenteral antimicrobial therapy for infections with methicillinsensitive Staphylococcus aureus. South Med J 2005;98:590-595.

11. Tokars JL, Cookson STM, Carther MA et al. Prospective evaluation of risk factors for bloodstream infection in patients receiving home infusion therapy. Ann Intern Med 1999;131:340-347.

12. Malani PN, Depestel DD, Riddle J et al. Experience with community-based amphotericin B infusion therapy. Pharmacotherapy 2005;25:690-697. 